\title{
Tarefas que avaliam a memória operacional na infância e adolescência: Uma revisão sistemática da literatura
}

\author{
Gustavo Marcelino Siquara \\ Universidade Estadual da Bahia \\ Escola Bahiana de Medicina e Saúde Pública \\ Maria Virgínia Machado Dazzani \\ Neander Abreu \\ Universidade Federal da Bahia
}

\begin{abstract}
Resumo
A Memória Operacional (MO) é um componente das funções executivas essenciais para o desenvolvimento cognitivo da criança. Ela tem efeitos sobre a aprendizagem e as tarefas diárias. O objetivo foi revisar a literatura para identificar as principais tarefas que foram utilizadas para avaliar a MO e a qualidade psicométrica. Foi realizada uma revisão da literatura em bancos de dados (Pubmed, Medline, Lilacs, SciELO). Foram analisados 44 artigos que utilizaram tarefas que avaliaram a alça fonológica e/ou esboço visuoespacial. A partir das análises dos artigos foram descritas um total de 20 diferentes tarefas para avaliar a MO. Com a revisão sistemática foi possível descrever diferentes tarefas a partir de um modelo cognitivo. No entanto, são necessários estudos psicométricos, para validação das medidas dentro do modelo teórico e o refinamento sobre o entendimento da MO.
\end{abstract}

Palavras-chave: memória operacional; infância; testes; função executiva.

\begin{abstract}
Tasks that assess working memory in children and adolescents: A systematic literature review. Working memory (WM) is an essential component of executive functions for the cognitive development of children. It has effects on learning and cognitive tasks daily. The objective was to review the literature to identify the key tasks that are being used to evaluate the WM and psychometric quality. A review of the literature databases (Pubmed, Medline, Lilacs, SciELO) was performed. Forty-four articles that presented tasks that assessed phonological and / loop or visuospatial sketch and described a total of 20 different task to assess WM were analyzed. It was possible to verify the existence of different tasks to assess WM. It is interesting to build different tasks from the model cognitive proposed. However, you must have theoretical foundation and psychometric studies for the proposed tasks.
\end{abstract}

Keywords: working memory; childhood; assessment; executive function.

\section{Resumen}

Tareas que evalúan la memoria de trabajo en niños y adolescentes: Una revisión sistemática de la literatura. La memoria de trabajo (MT) es un componente esencial de las funciones ejecutivas para el desarrollo cognitivo de los niños. Tiene efectos en las tareas de aprendizaje y de todos los días. El objetivo fue revisar la literatura para identificar las tareas clave que se utilizan para evaluar la calidad psicométrica y MT. Se realizó una revisión de las bases de datos bibliográficas (PubMed, Medline, Lilacs, SciELO). Cuarenta y cuatro artículos que utilizaron tareas que evaluaron fonológico y / o mango se analizaron boceto visuoespacial. A partir del análisis de los artículos se describieron un total de 20 tareas diferentes para evaluar la MT. Es interesante para construir diferentes tareas de un modelo cognitivo propuesto. Sin embargo, es necesario tener fundamento teórico y estudios psicométricos para las tareas propuestas.

Palabras clave: memoria de trabajo; infancia; las pruebas; función ejecutiva.

$\mathrm{A}$ Memória Operacional (MO) é a habilidade de armazenar e manipular a informação por um breve período de tempo (Kane \& Engle, 2002). Atrasos no desenvolvimento da MO podem fazer com que atividades diárias se tornem desafios. Uma vez que a MO tem a função de selecionar, analisar, conectar, sintetizar e resgatar as informações já apreendidas fazendo a conexão com informações novas (Lima, 2006). Prejuízos ou atrasos nesse subcomponente da função executiva podem estar associados a baixo desempenho escolar (Lima, 2006). O aparecimento do construto conhecido como "Memória 
Operacional" (Baddeley \& Hitch, 1974) refinou a compreensão sobre o funcionamento dos sistemas cognitivos, uma vez que o conceito único de memória de curto prazo, até então vigente, parecia não conseguir explicar uma série achados empíricos (Baddeley, 2011).

O modelo de MO que apresenta mais evidências neuropsicológicas e é o mais explorado na literatura é o Modelo Multicomponente de MO de Baddeley e Hitch (1974, 2000). Esse modelo é mais utilizado para explicar a $\mathrm{MO}$ e foi originalmente proposto com três componentes (Baddeley, 1986; Baddeley \& Hitch, 1974) atualmente é proposto com quatro componentes: o executivo central e três outros denominados escravos, a saber, a alça fonológica, o esboço visuoespacial e o retentor episódico (Baddeley, 2012).

Evidências de estudos com neuroimagem encontraram ativações em localizações distintas no cérebro durante a atividade da MO. Investigações neurológicas (Prabhakaran, Narayanan, Zhao, \& Gabrieli 2000) têm encontrado evidências de circuitos neurais separados para subcomponentes verbais e visuoespaciais. Outros estudos, a partir de modelos matemáticos, como o de Hedden e Yoon's (2006) indicaram que os componentes verbais, visuoespaciais e executivos da MO são associados com áreas distintas do cérebro.

Existem outros modelos para tentar explicar como a $\mathrm{MO}$ funciona (Cowan, 1995). Neste modelo, a ênfase é no foco atencional, em níveis de ativação e experiência, como propriedade essencial da MO. Cowan (1995) postula a interação estreita, mútua e a interdependência entre a MO e a Memória de Longo Prazo. Originalmente ele sugere que há um sistema de memória de armazenamento único que consiste em elementos com vários níveis de ativação (Cowan, 1995).

Nos últimos 20 anos, inúmeras pesquisas têm investigado a hipótese de que a MO é um processo que constitui a base da habilidade de aprender (Alloway \& Alloway, 2010; Alloway \& Passolunghi, 2011; Gathercole, Pickering, Knight, \& Stegmann 2004; Rohde \& Thompson, 2007; Zheng, Swanson, \& Marcoulides, 2011). A MO é requerida sempre que algo deve ser aprendido. A aprendizagem requer a manipulação da informação, interação com a memória de longo prazo e, simultaneamente, armazenamento e processamento da informação (Dehn, 2008). Existem evidências extensas na literatura de prejuízos associados a $\mathrm{MO}$ e diferentes quadros de transtornos que podem aparecer na infância e adolescência como TDAH, dislexia, síndromes genéticas, problemas de sono, depressão, esquizofrenia, transtorno bipolar, autismo, estresse, entre outros (Barendse, et al. 2013; Leutwyler et al. 2013; Mervis \& Velleman, 2011; Skogan, et al. 2013; Talarowska, Zboralski, \& Gałecki, 2013; Tucker, 2013; Wang \& Gathercole, 2013). A partir dos estudos citados, a MO parece ser uma função chave em muitos quadros que apresentam alterações no desenvolvimento. A partir do exposto ressalta-se a importância dentro da avaliação psicológica e enquanto construto que deve ser adequadamente estudado e investigado.

O desenvolvimento de modelos cognitivos deve ser construído e pautado com o maior número de evidências científicas.
Nos casos em que se pretende desenvolver uma medida (teste) que avalie uma função e/ou componente do modelo cognitivo é essencial a obtenção de dados empíricos e psicométricos. É preciso identificar se os dados gerados pelo instrumento que utilizamos baseado hipoteticamente em um modelo cognitivo são confirmados estatisticamente (Sternberg, 2009).

O que ocorre em alguns casos é que as tarefas utilizadas não passam pelo processo de validação e normatização formal que visa garantir a confiabilidade dos dados gerados. O processo de validação de instrumentos psicológicos se constitui em um processo mais geral, de validação de hipóteses científicas (Muniz, 2004). O processo de validação dos instrumentos que medem qualquer construto psicológico é fundamental para tentar assegurar a qualidade dos resultados gerados através dos testes. A validação dos testes envolve desafios metodológicos ao se deparar com a necessidade de estabelecer relações funcionais entre duas variáveis. Uma variável latente, o construto, e outra observada, os indicadores (Primi, 2010). Como a MO vem se tornando um construto cada vez mais importante, dentro do processamento cognitivo, é necessário conhecer e analisar a confiabilidades das medidas que se propõem a avaliar esse construto.

Os modelos da MO e, principalmente, o modelo multicomponente de Baddeley e Hitch (1974) e Baddeley (2000) têm sido amplamente utilizados nos estudos que investigam a capacidade de aprendizagem em crianças. As alterações em MO também são frequentes em síndromes genéticas, transtornos do desenvolvimento, entre outros.

Para conseguir fazer uma boa avaliação neuropsicológica e fazer dissociações funcionais é preciso conhecer e analisar a qualidade das diferentes tarefas (medidas) que avaliam a MO e seus componentes.

O objetivo desta investigação foi realizar uma revisão de literatura sobre as tarefas utilizadas para avaliar a alça fonológica e o esboço visuoespacial da MO na infância e adolescência. O outro objetivo foi analisar os estudos psicométricos referentes às tarefas encontradas utilizadas para avaliara MO.

\section{Material e método}

O estudo é caracterizado como uma revisão sistemática de artigos publicados em periódicos científicos nacionais e internacionais. Para a busca das publicações nas bases de dados foram utilizados os seguintes descritores: Working Memory, Executive Function and Child em inglês e Memória de Trabalho ou Memória Operacional em português. Os descritores foram escolhidos a partir da consulta de especialista na área.

Foram analisados os artigos publicados nos anos de 2008 a 2013, por se constituir as tarefas (medidas) mais recentes que foram utilizadas nos estudos sobre a MO. Os critérios de exclusão dos artigos para essa revisão foram: 1) estudos que não tenha utilizado tarefas neuropsicológicas que avaliem a alça fonológica e/ou esboço visuoespacial da MO. 2) estudos que não investigaram crianças e adolescentes ( 0 a 18 anos).

Os artigos foram buscados em cinco bases de dados sendo elas: Medline, Pubmed, Lilacs e SciELO. Essas bases de dados 
foram escolhidas por serem as principais bases de dados com revistas indexadas ao seu banco de dados na área do tema estudado.

Os dados foram retirados em uma folha de extração que registrou: 1) elenco dos estudos feitos por meio da identificação dos autores e ano de publicação; 2) descrição da tarefa; 3) componente da MO avaliado; 4) idade dos participantes; 5) estudo original que desenvolveu da tarefa.

\section{Resultados}

Foi encontrado um total de 245 artigos nos indexadores pesquisados (Medline, Pubmed, Lilacs e SciELO). Dos 245 artigos encontrados, foram eleitos 44 para serem analisados detalhadamente, de acordo com os critérios de exclusão.

As tarefas mais utilizadas para a avaliação da alça fonológica e o esboço visuoespacial da MO foram: a tarefa de amplitude de dígitos (digit span) da Escala Wechsler de Inteligência (WISC-III) e Cubos de Corsi (Corsi's Block-tapping), respectivamente, (Corso, 2012;
Coutinho, Mattos, \& Malloy-Diniz, 2009; Cowan, Saults, \& Morey, 2006; Franklin et al., 2010; Galera \& Souza, 2010; Göthe, Esser, Gendt, \& Kliegl, 2012; Grivol \& Hage, 2011; Iuculano, Moro, \& Butterworth, 2011; Rienstra, Spaan, \& Sch mand, 2010; Rodrigues \& Befi-lopes, 2009; Swanson, 2011; Vaz, Cordeiro, Macedo, \& Lukasova, 2010). Essas duas tarefas são apontadas na literatura como as mais clássicas e utilizadas para a avaliação dos componentes da MO e foram confirmadas a partir dessa revisão de literatura.

Dos 44 artigos analisados no total, foram encontradas 20 diferentes tarefas para avaliar a MO. Foram encontradas 20 tarefas, pois alguns dos 44 estudos analisados haviam utilizado a mesma tarefa (ver tabela 1). Dentro das tarefas descritas, nove avaliaram o esboço visuoespacial, nove a alça fonológica e dois avaliam ambos os componentes. A Tabela 1 mostra os componentes da $\mathrm{MO}$ avaliados pelas tarefas, nome da tarefa, a idade dos participantes do estudo, os estudos originais e o estudo que utilizou a tarefa nos últimos cinco anos.

Tabela 1

Tarefas que Avaliam Componentes da MO

\begin{tabular}{|c|c|c|c|c|}
\hline Componente & Teste & Idade & Estudo original & Estudos encontrados \\
\hline \multirow{9}{*}{ Esboço Visuoespacial } & Hide-and-seek & 18 meses & DeLoache, Cassidy, \& Brown (1985) & (Haden et al., 2011) \\
\hline & Spin thePots & 2 e 3 anos & Hughes \& Ensor, 2005 & (Matte-Gagné \& Bernier, 2011) \\
\hline & Knox's-cube teste & 6 anos & (Knox, 1914) & (Ni, Huang, \& Guo., 2011) \\
\hline & $\begin{array}{l}\text { Tarefa Matrix de Pontos - Automated } \\
\text { Working Memory Assessment (AWMA) }\end{array}$ & 6 a 16 anos & (Alloway, 2007) & (Dumontheil \& Klingberg, 2011; Ribeiro \& Santos, 2012) \\
\hline & Spatial Working Memory (CANTAB) & 6 a 18 anos & (Cambridge Cognition, 2006) & $\begin{array}{c}\text { (Franklin et al., 2010; Teixeira, Zachi, Roque, Taub, \& } \\
\text { Ventura, 2011) }\end{array}$ \\
\hline & Corsi's Block-tapping & 6 a 12 & (Milner, 1971) & (Franklin et al., 2010; Galera \& Souza, 2010) \\
\hline & Visual matrix task & 7 a 9 anos & (Swanson, 2011) & (Swanson, 2011) \\
\hline & Groton Maze Learning Test & 9 a 45 anos & (Pietrzak et al., 2008) & (Pietrzak et al., 2008) \\
\hline & $\begin{array}{l}\text { Memória sequencial não-verbal com } \\
\text { apoio visual }\end{array}$ & 9 a 11 anos & (Giangiacomo, \& Navas, 2008) & (Giangiacomo, \& Navas, 2008) \\
\hline \multirow{3}{*}{$\begin{array}{l}\text { Esboço Visuoespacial e } \\
\text { Alça Fonológica }\end{array}$} & Verbal-to-spatial mapping task & 9 a 13 anos & (Cowan et al., 2006) & (Ricker et al., 2010) \\
\hline & Imitation Sorting Task & 2 e 3 anos & (Alp, 1994) & (Fitzpatrick \& Pagani, 2012) \\
\hline & $\begin{array}{c}\text { Avaliação Automatizada de Memória } \\
\text { Operacional (AWMA) }\end{array}$ & 9 a 10 anos & (Alloway, 2007) & (Ribeiro, \& Santos, 2012) \\
\hline \multirow{8}{*}{ Alça Fonológica } & Digit Span & 10 a 12 anos & (Wechsler, 1997) & $\begin{array}{l}\text { (Imbo \& Vandierendonck, 2007); (Coutinho, Mattos, \& } \\
\text { Malloy-Diniz, 2009; Grivol \& Hage, 2011; luculano et } \\
\text { al., 2011; Vaz, Cordeiro, Macedo \& Lukasova, 2010) }\end{array}$ \\
\hline & Word Span & 8 e 9 anos & (luculano et al., 2011) & (luculano et al., 2011) \\
\hline & Listening sentence span & 7 a 9 anos & (Daneman \& Carpenter, 1980) & (Swanson, 2011) \\
\hline & Sentence/digit span & 7 a 9 anos & (Swanson, 2011) & (Swanson, 2011) \\
\hline & $\begin{array}{l}\text { Teste de memória sequencial auditi- } \\
\text { va de palavras e pseudopalavras }\end{array}$ & 9 a 11 anos & $\begin{array}{l}\text { (Navas, Ferraz, Giangiacomo, \& Satake, } \\
\text { 2005) }\end{array}$ & (Giangiacomo, \& Navas, 2008) \\
\hline & Tarefa N-Back Auditiva & 7 a 11 anos & (Kirchner, 1958) & (Nardi, et al. 2013) \\
\hline & Tarefa Listas de Palavras - Nepsy & 8 anos & Korkman, Kirk, Kemp, (1998) & (Nunest, et al. 2010) \\
\hline & Tarefa de Brown-Peterson & 6 a 11 anos & Peterson, Peterson (1959) & (Vaz, Cordeiro, Macedo, \& Lukasova, 2010) \\
\hline
\end{tabular}




\section{Descrição das tarefas}

As tarefas que se propõem avaliar a MO e presentes na Tabela 1 são descritas a seguir. O nome da tarefa é apresentado (primeira coluna), seguido do estudo original (quando encontrado/citado) e na última coluna, a referência do estudo que a utilizou a tarefa.

\section{Tarefas que avaliam o esboço visuoespacial}

Hide-and-seek (DeLoache, Cassidy, \& Brown,1985). O pesquisador acompanhado da criança esconde objetos familiares que são similares em forma e tamanho (por exemplo, boneco, bola) em vários locais, dentro de um quarto na casa da criança (por exemplo, embaixo de um travesseiro, atrás de uma cortina). Após um período de intervalo, a criança é incentivada a procurar cada objeto até que todos sejam encontrados (Haden et al., 2011).

Spin the pots (Hughes \& Ensor, 2005). Nesta tarefa, as crianças são orientadas a procurar objetos que são apresentados. Os objetos são cobertos em potes opacos, que são girados, modificando a posição dos mesmos. Seis objetos e oito potes de diferentes aparências visuais foram utilizados (Matte-Gagné \& Bernier, 2011).

Knox's-cube teste (Knox, 1914). A tarefa é constituída por uma fileira alinhada de quatro cubos (de uma polegada) cada um, enfileirados (distância de quatro polegadas) e presos a uma base. $\mathrm{O}$ examinador toca em uma sequência de cubos. A tarefa do experimentando é tocar a mesma ordem de imediato. A outra tarefa é tocar os cubos na ordem inversa. Na ordem inversa o Teste de Knox é usado como uma medida da MO para o esboço visuoespacial (Ni, Huang, \& Guo, 2011).

Tarefa matriz de pontos - Automated Working Memory Assessment (Alloway, 2007). A tarefa visual envolve lembrar o local e a ordem de pontos exibidos em sequência de uma grade na tela do computador. No teste, a dificuldade foi aumentada após quatro ensaios respondidos corretamente, adicionando um item a ser lembrado. $O$ teste encerra quando três erros são cometidos em um nível. (Dumontheil \& Klingberg, 2011).

Spatial working memory - Cambridge Neuropsychological Test Automated Battery (CANTAB) (Cambridge Cognition, 2006). A tarefa é uma busca auto ordenada de estímulos espaciais e exige que o sujeito use a informação mnemônica para alcançar um objetivo. Os experimentandos são solicitados a buscar fichas azuis dentro de caixas que aparecem na tela de um computador. A instrução chave é que uma vez que uma ficha tenha sido encontrada dentro de uma caixa, a caixa não será utilizada novamente. Pelo processo de eliminação, o sujeito deve encontrar as fichas azuis e utilizá-las para encher uma coluna vazia à direita na tela do computador. O sujeito decide a ordem em que as caixas são visitadas. É considerado erro voltar a uma caixa, na qual já tenha encontrado a ficha azul (Franklin et al., 2010).

Corsi's block-tapping (Milner, 1971). A tarefa consiste de nove cubos montados em uma plataforma. Na primeira tarefa o examinador toca nos blocos e o examinando toca na mesma sequência, formando a ordem direta. Na segunda tarefa, o examinando deve tocar os cubos na ordem inversa. Esta tarefa mede a capacidade de recordar uma sequência de cubos apresentados na plataforma (Franklin et al., 2010).

Visual matrix task (Swanson, 2011). A finalidade desta tarefa é avaliar a capacidade dos experimentandos para lembrar sequências visuais dentro de uma matriz. Aos examinandos é apresentada uma série de pontos de uma matriz com um intervalo de 5 segundos para estudar a matriz. Está é removida e pergunta-se aos experimentandos: "Existem pontos na primeira coluna?" Depois de responder a questão discriminatória, os examinandos são convidados a desenhar os pontos recordados da matriz nas caixas correspondentes de seus livretos de resposta. A dificuldade da tarefa varia de uma matriz de quatro quadrados (conjunto 1 ) e dois pontos a uma matriz de 45 quadrados e 12 pontos (conjunto 11) (Swanson, 2011).

Groton Maze Learning Test (Pietrzak et al., 2008). Consiste em 100 quadrados (matriz 10x10) apresentados em uma tela de computador sensível ao toque. Para completar a tarefa, o experimentando tem que seguir um caminho oculto (28 movimentos) através dos quadrados a partir do canto superior esquerdo, para um sinal no canto inferior direito. Barras com mensagem na parte superior e inferior da tela informam ao experimentando se o movimento está correto. Se o movimento estiver correto, uma mensagem e um som são apresentados no qual está escrito "Go On" (continue). Se o movimento estiver incorreto, ele é registrado como um "erro" e o experimentando é instruído a voltar para a última posição correta e tentar mover em uma direção diferente. Se duas respostas incorretas são emitidas em uma fileira, o segundo erro consecutivo é rotulado como um "erro perseverante" e o experimentando é novamente instruído a voltar para o quadrado correto, anterior, e tentar um novo caminho. Se o experimentando não voltar para o último quadrado correto depois de dois movimentos sucessivos errados (ou seja, três vezes jogadas erradas consecutivas), o terceiro erro é rotulado como um erro de quebra de regra e os quadrados que correspondem ao último movimento correto começam a piscar. O experimentando é, então, solicitado a tocar em um local específico da tela e então continuar através do labirinto (Pietrzak et al., 2008).

Teste de memória sequencial não-verbal com apoio visual. É realizado como um jogo que produz diferentes sons em sequência ao mesmo tempo em que as luzes dos correspondentes se acendem. A sequência inicia-se com um estímulo e a cada acerto, um novo estímulo é acrescentado à sequência anterior. Se houver erro, o jogo se encerra. Para a avaliação da memória não-verbal é estabelecido o seguinte critério: foram três tentativas e o nível de memória não-verbal foi determinado pela média de acertos (Giangiacomo \&Navas, 2008).

\section{Tarefas que avaliam o esboço visuoespacial e alça fonológica}

Avaliação Automatizada de Memória Operacional (AWMA) (Alloway, 2007). É um teste computadorizado que abrange a avaliação tanto da capacidade de armazenamento, processamento 
de informações e operações avaliadas de forma balanceada por tarefas na modalidade verbal e visuoespacial. A bateria possui 12 subtestes, cada um composto por um número variado de séries, com sequências que aumentam a complexidade progressivamente (Ribeiro \& Santos, 2012).

\section{Tarefas que avaliam conjuntamente o esboço vi- suoespacial e alça fonológica}

Verbal-to-spatial mapping task. Nesta tarefa, aos experimentandos são mostrados os locais de alguns rótulos verbais e em seguida, após um pequeno intervalo, deve-se colocar um desses rótulos verbais no local correto. Os rótulos verbais foram dois conjuntos de palavras comuns e fonologicamente distintas. Um era o conjunto de nomes de meninas: Ann, Beth, Graça, Dawn, Ruth, Eva, Liz, Marge, e Joy, e o outro conjunto consistia de nomes de meninos: Bob, Dan, Joe, Mike, Ken, Chuck, Will, Steve, e Ray (Ricker, Cowan, \& Morey, 2010).

Imitation Sorting task (Alp, 1994). O objetivo da tarefa é que as crianças reproduzam corretamente a sequência demonstrada, através da colocação do brinquedo correto na lata correta. No início de cada ensaio, o examinador coloca brinquedos na frente da criança. Em seguida, nomeia cada brinquedo, atraindo a atenção da criança antes de colocá-lo em uma lata. O procedimento é repetido para os outros brinquedos. Os brinquedos são então removidos das latas e colocados em frente à criança. O examinador, em seguida, pede à criança para colocar os objetos nas mesmas latas. Em cada nível de dificuldade a criança tem uma ordem para colocar corretamente os objetos nas latas (Fitzpatrick \& Pagani, 2012).

\section{Tarefas que avaliam a alça fonológica}

Digit span (Wechsler, 1997). Os estímulos utilizados são dígitos numéricos aleatórios que são verbalizados com 1 segundo de intervalo entre os dígitos. Para cada item de Dígitos na Ordem Direta, a criança repete os números na mesma ordem em que foram falados. Para cada item de Dígitos na Ordem Inversa, a criança repete os números na ordem inversa. Cada item apresenta duas tentativas e cada tentativa possui o mesmo número de dígitos, mas os números são diferentes (Iuculano et al., 2011).

Word span. Os estímulos da tarefa são palavras em duas categorias semânticas - Animais e Objetos - e foram selecionados a fim de corresponder aos estímulos em número de sílabas (1-5) e comprimentos de palavra (número de letras). As listas são verbalizadas de acordo com o nível de complexidade crescente, de dois a nove itens. Os estímulos são verbalmente apresentados pelo examinador a uma taxa de um item por segundo (Iuculano et al., 2011).

Listening sentence span (Daneman \& Carpenter, 1980). Esta tarefa exige a apresentação de grupos de frases, lidas em voz alta, para que as crianças ao mesmo tempo entendam o conteúdo da sentença e lembrem-se da última palavra de cada frase. O número de frases no grupo gradualmente aumenta de 2 a 6. Depois que cada grupo(s) de frase(s) foi apresentado, o experimentando responde a uma pergunta sobre a(s) frase(s) e então é convidado a recordar a última palavra de cada frase(s) (Swanson, 2011).

Sentence/digit span. Esta tarefa avalia a capacidade da criança para lembrar sequências numéricas embutidas em uma frase curta. Por exemplo: "Suponha que alguém queira levá-lo ao supermercado às 8651 na Elstreet”. Os números são apresentados em intervalos de 2 segundos, seguido por uma questão de processo, ou seja, "Qual era o nome da rua?". A variável dependente foi o número total de itens (dígitos) corretos até o maior conjunto. O tamanho do conjunto variou de dois a 14 dígitos (Swanson, 2011).

Testes de Memória Sequencial Auditiva de Palavras e Pseudopalavras (Navas, Ferraz, Giangiacomo, \& Satake, 2005). O teste contém 60 estímulos cada um. Todos os estímulos são dissílabos paroxítonos com estrutura Consoante-Vogal-Consoante-Vogal. São apresentadas quatro listas com três blocos de estímulos com duas, três, quatro, cinco e seis sequências de palavras (L3 e L4) e pseudopalavras (L1 e L2) em cada bloco. O intervalo entre os estímulos é de 1 segundo para as listas L1 e L4 e de 500 milisegundos para as listas L2 e L3. A apresentação é feita por meio de $C D$ player com fones de ouvido e as respostas são gravadas. É solicitado que o testando ouça a sequência de estímulos de um bloco e ao final repeti-los na ordem correta (Giangiacomo \& Navas, 2008).

$N$-Back auditiva (Kirchner, 1958). Nessa tarefa os participantes escutam uma sequência de números, apresentados um por segundo, enquanto escutam cada número, têm de dizer o que ocorreu "N" (1-back; 2-back; 3-back, etc) posições atrás na série, por recuperação livre e sem pistas. Condição "0-Back”, o examinador diz um número e o testando deve repeti-lo imediatamente depois. Por exemplo: se eu disser "7" você deve dizer "7", e assim seguimos a tarefa. Condição "1 back" Quando o examinador disser um número, o testando deve dizer o número imediatamente anterior. Por exemplo, se o examinador falar a série de dígitos “ $3,2,9,4,2 .$. ", o testando deverá falar imediatamente após o primeiro dígito, no caso "2", a palavra "nenhum”. Em seguida, após ouvir “2”, deverá dizer “3”, exatamente o dígito anterior. Na sequência, portanto, após ouvir “9”, deverá dizer " 2 ", após ouvir " 4 " deverá dizer "9" e assim por diante". "Logo após o "3”, você deve dizer "nenhum”, porque não tinha nenhum número antes, certo? E assim segue a sequência com "2 back”, “3 back” (Nardi, et al. 2013).

Tarefa listas de palavras (NEPSY-III) (Korkman, Kirk, \& Kemp, 1998). Este subteste foi elaborado para avaliar a MO verbal, repetição e recordação de palavras seguintes à interferência. São apresentadas aos examinandos duas séries de palavras e solicitado que repita cada sequência após a apresentação. Depois, o examinando deve recordar cada série na ordem de apresentação (Nunest, 2010).

Tarefa de Brown-Peterson (Peterson \& Peterson, 1959). São compostas por 20 itens, sendo que cada item é um conjunto de três consoantes diferentes com baixa similaridade fonológica. Os itens são divididos em quatro conjuntos: parte I do teste contém um conjunto de itens para evocação imediata e parte II do teste contém três conjuntos para a evocação com interferência. $\mathrm{Na}$ 
evocação imediata (parte I), o participante é solicitado a escutar atentamente três letras diferentes e repeti-las imediatamente após a apresentação. São apresentadas cinco tríades de letras. Já na evocação com interferência (parte II), o participante é solicitado a repetir a tríade de letras somente após um intervalo de tempo, durante o qual ele realizará uma tarefa de contar a partir de um número oferecido pelo aplicador sendo esta uma interferência verbal. O intervalo de tempo é de 3s, 9s ou $18 \mathrm{~s}$. Na parte II, há 15 itens ao todo, sendo cinco itens para cada intervalo de atraso com apresentação randomizada. A pontuação final pode ser de até 60 pontos, e é a soma das letras corretamente evocadas (Vaz, Cordeiro, Macedo, \& Lukasova, 2010).

\section{Discussão}

A presente revisão encontrou um total de 20 tarefas que avaliam componentes da MO, segundo o modelo multicomponente de Baddeley e Hitch (1974) e Baddeley (2000). Entre as tarefas encontradas, nove avaliam o esboço visuoespacial, três o esboço visuoespacial e a alça fonológica simultaneamente e nove a alça fonológica. As tarefas mais citadas foram amplitude de dígitos (digit span) para avaliar a alça fonológica e a tarefa Cubos de Corsi (Corsi Block Test) para avaliar o esboço visuoespacial. Nessa revisão foram encontrados estudos que utilizaram tarefas para avaliar crianças desde os 18 meses até os 16 anos de idade.

Foi possível identificar e descrever diferentes tarefas que avaliam os componentes da MO em crianças e adolescentes. Essas tarefas apresentam diferentes paradigmas de manipulação da informação mental, que pode ser através de um input verbal (alça fonológica) ou visual-espacial (esboço visuoespacial). Algumas tarefas recrutam a manipulação da informação como o Groton Maze Learning Test e outras requisitam menos, como o Automated Working Memory Assessment. Outro exemplo é a tarefa de amplitude de palavras (Word Span), na qual a pessoa deve apenas lembrar as palavras ou no Listening sentence span em que requisita apenas a lembrança da amplitude auditiva de sentenças na qual o testando deve entender a frase e lembrar-se da última palavra. Algumas tarefas apresentam uma demanda de processamento maior do que outras, e isso, provavelmente influencia os resultados. A possibilidade de desenvolver tarefas a partir do modelo de $\mathrm{MO}$ é interessante e corrobora o caráter funcional da MO, no entanto é preciso analisar os dados psicométricos dessas tarefas. Isso é interessante, pois diferentes tarefas apresentam formas e intensidade da manipulação da informação na MO de maneira mais acentuada, o que pode influenciar nos resultados, na interpretação dos resultados e na atribuição do modelo cognitivo. O uso de um modelo cognitivo para a compreensão da função é fundamental porque permite desenvolver diferentes tarefas, testar hipóteses e estabelecer dissociações com áreas e funções cerebrais. A utilização de um modelo cognitivo requer também que o dado empírico gerado pelo instrumento seja válido, pois assim será possível dizer se o instrumento avalia a MO.

O cuidado com a qualidade dos dados gerados nos estudos deve ser tratado como prioridade. Um dos exemplos de falhas metodológicas é o estudo original de Haden et al. (2011) e a tarefa "Hide-and-seek". Essa tarefa foi descrita no seu estudo original (DeLoache, Cassidy, \& Brown 1985) como uma medida rudimentar e imperfeita sobre o que irá se tornar uma estratégia de processamento mnemônico. Por outro lado, no estudo investigado Haden et al. (2011) descrevem a mesma tarefa como suficiente para avaliar a MO. A tarefa, como foi apresentada, se refere a uma proposta de localização de objetos, não havendo a manipulação de informação mental. É possível que haja realmente uma falha teórico-metodológica ao utilizar essa tarefa para avaliação da MO. A tarefa parece efetivamente avaliar o desempenho da memória, de uma forma geral, sem especificar qualquer componente da memória.

Matte-Gagné e Bernier (2011) e Fitzpatrick e Pagani (2012) utilizam tarefas que acreditam avaliar a $\mathrm{MO}$ em crianças de 2 a 3 anos na tarefa "Spin the Pots" e "Imitation Sorting Task", respectivamente. De acordo com a descrição, na tarefa ocorre o armazenamento de curto prazo, mas novamente parece não ocorrer o processo de manipulação mental, característico da MO. Outra questão é que nesses estudos é necessário enfatizar as limitações em avaliar a MO em crianças tão pequenas. Alloway, Gathercole, Willis e Adams, (2004) defendem que a MO possa ser avaliada de forma confiável a partir de 4 anos de idade. Antes dos 4 anos talvez seja possível analisar aspectos rudimentares da $\mathrm{MO}$, sendo talvez um primeiro indício do desenvolvimento. As limitações dos instrumentos e possíveis generalização dos resultados, principalmente nos estudos que se propõem a avaliar crianças menores de 4 anos, devem ser enfatizadas. Estudos que utilizem essas tarefas parecem depender de dados psicométricos mais refinados para poder afirmar que estão avaliando a memória de MO.

Estudos com crianças têm sugerido que a MO e a Memória de Curto Prazo (MCP) são pouco distintas em crianças jovens em comparação a crianças mais velhas ou em adultos (Alloway, Gathercole, \& Pickering 2006; Engel de Abreu, Conway, \& Gathercole, 2010). Por exemplo, o estudo de Alloway et al., (2006) sobre a diferenciação entre a MCP e MO em crianças, apontou que esses construtos podem ser mais sobrepostos dependendo da fase de desenvolvimento que o indivíduo se encontra. Em crianças, a distinção entre construtos parece ser influenciada bastante pela fase do desenvolvimento. Isso provavelmente ocorre pela fase do desenvolvimento como também parece associada ao desenvolvimento de sistemas cerebrais e cognitivos, que se tornam progressivamente mais especializados e melhor detectados por técnicas psicométricas. A formação dos sistemas cognitivos parece depender da fase do desenvolvimento. Com isso deve-se ter um cuidado maior com dados gerados por instrumentos que avaliam um componente cognitivo sem passar por um rigoroso controle de validação das medidas.

Outro dado encontrado com a pesquisa foi a falta de referência dos estudos, aos dados de validação e normatização dos instrumentos que são utilizados. Especificamente para o contexto brasileiro, apenas a tarefa de Amplitudes de Dígitos (digit span) apresenta estudo de validação e normatização. Nenhum outro estudo encontrado, nesta revisão, apresenta dados de validação e/ou normatização para o contexto brasileiro. Apenas o estudo de 
Ribeiro e Santos, (2012) cita um estudo de adaptação de Santos e Engel (2008) para a tarefa Automated Working Memory Assessment. Nem mesmo a tarefa de Cubos de Corsi (Milner, 1971), que é uma das mais utilizadas na literatura brasileira, apresenta estudo de validação para o contexto brasileiro. Alguns estudos encontrados tiveram o objetivo de avaliar a $\mathrm{MO}$ e identificar as diferenças entre diferentes faixas etárias (Nardi et al., 2013), a relação com a leitura (Giangiacomo \& Navas, 2008) entre crianças e adolescentes (Teixeira, et al., 2011), no entanto não propõe, citam ou apresentam dados dos estudos de validação ou indícios da mesma. Seria interessante que nesses estudos fossem apresentados dados psicométricos que demonstrassem a análise do construto proposto. Ou seja, não foi encontrado na presente revisão instrumentos, no Brasil, para avaliar a $\mathrm{MO}$ que apresentem dados de validação no contexto nacional com exceção da tarefa de amplitudes de dígitos.

A investigação da $\mathrm{MO}$ e seus componentes, a partir de diferentes tarefas, contribuem de maneira direta para o diagnóstico diferencial em neuropsicologia, identificação dos fenótipos cognitivos e possíveis relações com a aprendizagem. Alguns trabalhos já investigaram a $\mathrm{MO}$ e as relações com a aprendizagem como nos Transtorno do Déficit de Atenção/ Hiperatividade (TDAH) (Alloway, Elliot, \& Homes 2010), dislexia (Alloway \& Archibald, 2008), discalculia (Bird, 2009), transtorno invasivos do desenvolvimento (Alloway, Rajendran, \& Archibald, 2009). É importante que esses estudos estejam presentes também no contexto brasileiro para que se possam ter mais ferramentas para a investigação da relação entre $\mathrm{MO}$ e a aprendizagem, mas com a apresentação dos dados psicométricos. A descrição e apresentação de diferentes tarefas nesse artigo propõe chamar atenção para a importância de traduzir, validar e desenvolver novas tarefas para avaliar a MO dentro do contexto brasileiro. É necessário desenvolver medidas que sejam validadas empiricamente, para poder garantir a qualidade dos dados gerados com a medida e o avanço do conhecimento na área. Essas medidas utilizadas precisam ter evidências do construto que elas avaliam, para possibilitar a comparação entre resultados e os estudos tenham um maior impacto e contribuição para a ciência.

O modelo multicomponente da MO (Baddeley, 2000; Baddeley \& Hitch, 1974) parece facilitar o desenvolvimento de tarefas para avaliação de seus componentes, por ser um modelo relativamente simples e fácil de entender. Por um lado, isso é muito proveitoso, pois é possível elaborar diferentes tarefas para avaliar o mesmo construto. Existe, entretanto, um risco a nível teórico e metodológico de que uma tarefa que, aparentemente, está medindo um componente da MO possa medir conjuntamente outros componentes e com erro. Com isso resulta na falta do controle dos resultados que o instrumento produz. Por isso é necessário desenvolver procedimentos psicométricos controlados para que se possa usar a tarefa como teste e investigar sua validade. Utilizar tarefas com manipulação mental, graus de demanda para essa manipulação, diferentes estímulos; mas, para isso, as medidas precisam ser validas. A partir do momento que isso ocorrer, irá facilitar a comparação entre os resultados encontrados com o avanço no conhecimento sobre MO. A utilização de diferentes instrumentos para medir o mesmo construto, submetidos a processos de validade pode trazer boas contribuições para a área da avaliação da MO.

Para concluir, o desenvolvimento de diferentes tarefas para avaliar a MO é muito interessante do ponto de vista da avaliação neuropsicológica. No entanto, se corre um risco conceitual e metodológico quando se utiliza de tarefas que não passaram pelo processo de validação. O que se encontra são tarefas com diferentes níveis de dificuldade, envolvido com diferentes aspectos, só que não se encontra uma solidez conceitual das tarefas para trazer evidências daquilo que ela está se propondo a medir. Pressupostos teóricos e dados psicométricos para a utilização das tarefas em estudos de avaliação de MO infantil devem ser cuidadosamente desenvolvidos, revistos e atualizados. Sugere-se investigar as tarefas utilizadas para a avaliação da $\mathrm{MO}$ e sua adequação aos modelos cognitivos para que se escolha de maneira correta as tarefas a serem utilizadas nos estudos. Resultados de pesquisas ou clínicos sem uma explicação cognitiva é como um carro sem motor. Os dados puramente empíricos, sem uma explicação cognitiva, não representam contribuições para o desenvolvimento da área. Para tanto são necessários estudos adicionais que investiguem a adequação das tarefas ao modelo de $\mathrm{MO}$, seus limites e potenciais quando utilizados com crianças.

\section{Referências}

Alloway, T. P. (2007). Automated working memory assessment manual. Oxford: Harcourt.

Alloway, T. P., \& Alloway, R. G. (2010). Investigating the predictive roles of working memory and IQ in academic attainment. Journal of Experimental Child Psychology, 106(1), 20-29. doi:10.1016/j.jecp.2009.11.003

Alloway, T. P., \& Archibald, L. M. (2008). Working memory and learning in children with developmental coordination disorder and specific language impairment. Journal of Learning Disabilities, 41, 251-262. doi: $10.1177 / 0022219408315815$

Alloway, T. P., \& Passolunghi, M. C. (2011).The relationship between working memory, IQ, and mathematical skills in children. Learning and Individual Differences, 21(1), 133-137. doi: 10.1016/j.lindif.2010.09.013

Alloway, T. P., Gathercole, S. E., \& Pickering, S. J. (2006). Verbal and visuospatial short-term and working memory in children: are they separable? Child Development, 77(6), 1698-716. Retirado de http://www.york.ac.uk/res/ wml/Alloway,\%20Gathercole\&Pickering06.pdf

Alloway, T. P., Elliot, J., \& Homes, J. (2010). The prevalence of ADHD like symptoms in a community sample. Journal of Attention Disorders, 14, 5256. doi: $10.1177 / 1087054709356197$

Alloway, T. P., Gathercole, S. E., Adams, A. M., Willis, C., Eaglen, R., \& Lamont, E. (2005). Working memory and phonological awareness as predictors of progress towards early learning goals at school entry. British Journal of Developmental Psychology, 23, 417-426. doi: 10.1348/026151005X26804

Alloway, T. P., Gathercole, S. E., Willis, C., \& Adams, A. M. (2004). A structural analysis of working memory and related cognitive skills in early childhood. Journal of Experimental Child Psychology, 87, 85-106. doi: 10.1016/j. jecp.2003.10.002

Alloway, T. P., Rajendran, G., \& Archibald, L. M. (2009). Working memory profiles of children with developmental disorders. Journal of Learning Difficultes, 42, 372-382. doi: 10.1177/0022219409335214

Alp, I. E. (1994). Measuring the size of working memory in very young children: The imitation sorting task. International Journal of Behavioral Development, 17, 125-141. doi: 10.1177/016502549401700108 
Baddeley, A. D. (1986). Working memory. Nova Iorque: Oxford University Press.

Baddeley, A. D. (1996). Exploring the central executive. Quarterly Journal of Experimental Psychology, 49(1), 5-28. doi:10.1080/713755608

Baddeley, A. D. (2000). The episodic buffer: A new component of working memory? Trends in Cognitive Sciences, 4(11), 417-423. doi:10.1016/S13646613(00)01538-2

Baddeley, A. D. (2011). Memória de trabalho. In A. D. Baddeley, M. W. Eysenck, \& M. C. Anderson (Orgs.), Memória (pp. 54-82). Porto Alegre: Artmed.

Baddeley, A. D. (2012). Working memory: Theories, models, and controversies. Annual Review of Psychology, 63, 1-29. doi: 10.1146/annurevpsych-120710-100422

Baddeley, A. D., \& Hitch, G. J. (1974). Working memory. In G. H. Bower (Org.), The psychology of learning and motivation (pp. 47-90). Londres: Academic Press.

Baddeley, A. D., \& Hitch, G.J. (2000). Development of working memory: Should the Pascual-Leone and the Baddeley \& Hitch models be merged? Journal of Experimental Child Psychology, 77, 128-137.

Barendse, E. M., Hendriks, M. P., Jansen, J. F., Backes, W. H., Hofman, P. A., Thoonen, G.; ... Aldenkamp, A. P. (2013). Working memory deficits in high functioning adolescents with autism spectrum disorders: Neuropsychological and neuroimaging correlates. Journal Neurodevelopment Disorders, 5(1), 14. doi: 10.1186/1866-1955-5-14

Bird, R. (2009). Overcoming difficulties with number. Londres: Sage.

Cambridge Cognition (2006). Neuropsychological Test Automated Battery (CANTAB) manual. Cambridge: Cambridge Cognition Limited.

Corso, L. V. (2012). Qual o papel que a memória de trabalho exerce na aprendizagem da matemática? Bolema, 26(42B), 627-647. doi: 10.1590/ S0103-636X2012000200011

Cowan, N. (1995). Attention and memory: An integrated framework (Oxford psychology series). Nova Iorque: Oxford University Press.

Coutinho, G., Mattos, P., \& Malloy-Diniz, L. F. (2009). Neuropsychological differences between attention deficit hyperactivity disorder and control children and adolescents referred for academic impairment. Revista Brasileira de Psiquiatria (São Paulo), 31, 141-144.

Cowan, N., Saults, J. S., \& Morey, C. C. (2006). Development of working memory for verbal-spatial associations. Journal of Memory and Language, 55(2), 274289. doi:10.1016/j.jml.2006.04.002

Daneman, M., \& Carpenter, P. A. (1980). Individual differences in working memory and reading. Journal of Verbal Learning and Verbal Behavior, 19, 450-466. doi: 10.1016/S0022-5371(80)90312-6

Dehn, M. J. (2008). Working memory and academic learning: Assessment and intervention. Hoboken, NJ: Jonh Wiley \& Sons.

DeLoache, J. S., Cassidy, D. J., \& Brown, A. L. (1985). Precursors of mnemonic strategies in very young children's memory. Child Development, 56, 125137. doi: 10.1111/j.1467-8624.1985.tb00092.x

Dumontheil, I., \& Klingberg, T. (2011). Brain activity during a visuospatial working memory task predicts arithmetical performance 2 years later. Cerebral Cortex, 22, 1078-1085. doi:10.1093/cercor/bhr175

Engel de Abreu, P. M. J, Conway, A. R. A., \& Gathercole, S. E. (2010). Working memory and fluid intelligence in young children. Intelligence, 38(6), 552561. doi: 10.1016/j.intell.2010.07.003

Fitzpatrick, C., \& Pagani, L. S. (2012). Toddler working memory skills predict kindergarten school readiness. Intelligence, 40(2), 205-212. doi:10.1016/j. intell.2011.11.007

Franklin, T., Lee, A., Hall, N., Hetrick, S., Ong, J., Haslam, N., ... Vance, A. (2010). The association of visuospatial working memory with dysthymic disorder in pre-pubertal children. Psychological Medicine, 40(2), 253-61. doi:10.1017/S0033291709990365

Galera, C., \& Souza, A. L. P. (2010). Memória visuoespacial e cinestésica de curto prazo em crianças de 7 a 10 anos. Estudos de Psicologia, 15(2), 137-143. doi 10.1590/S1413-294X2010000200002
Gathercole, S. E., Pickering, S. J., Knight, C., \& Stegmann, Z. (2004).Working memory skills and educational attainment: Evidence from national curriculum assessments at 7 and 14 years of age. Applied Cognitive Psychology, 18, 1-16. doi: 10.1002/acp.934

Giangiacomo, M. C. P. B., \& Navas, A. L. G. P. (2008) . A influência da memória operacional nas habilidades de compreensão de leitura em escolares de $4^{\text {a }}$ série. Revista Sociedade Brasileira Fonoaudiologia, 13(1), 69-74. doi: 10.1590/ S1516-80342008000100012

Göthe, K., Esser, G., Gendt, A., \& Kliegl, R. (2012). Working memory in children: Tracing age differences and special educational needs to parameters of a formal model. Developmental Psychology, 48(2), 459-76. doi: 10.1037/ $\mathrm{a} 0025660$

Grivol, M. P., \& Hage, S. R. V. (2011). Memória de trabalho fonológica: Estudo comparativo entre diferentes faixas etárias. Jornal da Sociedade Brasileira Fonoaudiologia. 23(3), 245-51. doi: 10.1590/S2179-64912011000300010

Haden, C. A, Ornstein, P. A, O’Brien, B. S., Elischberger, H. B., Tyler, C. S., \& Burchinal, M. J. (2011). The development of children's early memory skills. Journal of Experimental Child Psychology, 108(1), 44-60. doi:10.1016/j. jecp.2010.06.007

Hedden, T., \& Yoon, C. (2006). Individual differences in in executive processing predict susceptibility to interference in verbal working memory. Neuropsychology, 20, 511-528. doi: 10.1037/0894-4105.20.5.511

Hughes, C., \& Ensor, R. (2005). Executive function and theory of mind in 2-year-olds: A family affair? Developmental Neuropsychology, 28, 645-668. doi: 10.1207/s15326942dn2802_5

Imbo, I., \& Vandierendonck, A. (2007). The development of strategy use in elementary school children: working memory and individual differences. Journal of Experimental Child Psychology, 96(4), 284-309. doi:10.1016/j. jecp.2006.09.001

Iuculano, T., Moro, R., \& Butterworth, B. (2011). Updating working memory and arithmetical attainment in school. Learning and Individual Differences, 21(6), 655-661. doi:10.1016/j.lindif.2010.12.002

Kane, M. J., \& Engle, R. W. (2002). The role of prefrontal cortex in workingmemory capacity, executive attention, and general fluid intelligence: An individual differences perspective. Psychonomic Bulletin and Review, 9, 637671. doi: 10.3758/BF03196323

Kirchner, W. K. (1958). Age differences in short-term retention of rapidly changing information. Journal of Experimental Psychology, 55(4), 352-358. doi: $10.1037 / \mathrm{h} 0043688$

Knox, H. (1914). A scale, based on the work at Ellis Island, for estimating mental defect. Jama, 62(10), 741. doi:10.1001/jama.1914.02560350001001

Korkman, M., Kirk, U., \& Kemp, S. (1998). NEPSY: A Developmental neuropsychological assessment. San Antonio, TX: The Psychological Corporation.

Leutwyler, H., Hubbard, E. M., Jeste, D. V., Miller, B., \& Vinogradov, S. (2013). Associations of schizophrenia symptoms and neurocognition with physical activity in older adults with schizophrenia. Biology Research Nursey, 16(1), 23-30. doi: 10.1177/1099800413500845.

Matte-Gagné, C., \& Bernier, A. (2011). Prospective relations between maternal autonomy support and child executive functioning: Investigating the mediating role of child language ability. Journal of Experimental Child Psychology, 110(4), 611-25. doi:10.1016/j.jecp.2011.06.006

Mervis, C. B., \& Velleman, S. L. (2011). Children with Williams Syndrome: Language, cognitive, and behavioral characteristics and their implications for intervention. Perspective Lang Learning Education; 18(3), 98-107. doi: 10.1044\%2Flle18.3.98

Milner, B. (1971). Interhemispheric differences in the localisation of psychological processes in man. British Medical Bulletin, 27, 272-277.

Muniz, J. (2004). La validación de los tests. Metodología de las Ciencias del Comportamiento, 5(2), 121-141.

Nardi, T., Sanvicente-Vieira, B., Prando, M., Stein, L. M., Fonseca, R. P., \& GrassiOliveira, R. (2013). Tarefa N-back auditiva: Desempenho entre diferentes grupos etários. Psicologia: Reflexão e Crítica, 26(1), 151-159. doi: 10.1590/ S0102-79722013000100016 
Ni, T.-L., Huang, C.-C., \& Guo, N.-W. (2011). Executive function deficit in preschool children born very low birth weight with normal early development. Early Human Development, 87(2), 137-41. doi: 10.1016/j. earlhumdev.2010.11.013

Nunest, S., Miranda, D. L., Reis, A. T., Gramacho, A. M. S., Lucena, R., \& Argollo, N. (2010). Complicações neurológicas em anemia falciforme: avaliação neuropsicológica do desenvolvimento com o NEPSY. Revista Brasileira de Hematologia e Hemoterapia, 32(2), 181-185. doi: 10.1590/S151684842010005000044

Peterson, L., \& Peterson, M. (1959). Short-term retention of individual verba items. Journal of Experimental Psychology, 58(3), 193-198. doi: 10.1037/ h0049234

Pietrzak, R. H., Maruff, P., Mayes, L. C., Roman, S. A., Sosa, J. A., \& Snyder, P. J. (2008). An examination of the construct validity and factor structure of the groton maze learning test, a new measure of spatial working memory, learning efficiency, and error monitoring. Archives of Clinical Neuropsychology, 23(4), 433-45. doi:10.1016/j.acn.2008.03.002

Prabhakaran, V.; Narayanan, K.; Zhao, Z., \& Gabrieli, J. D. E. (2000). Integration of diverse information in working memory within the frontal lobe. Nature Neurosciense, 3(1), 85-90. doi:10.1038/71156

Primi, R. (2010). Avaliação psicológica no Brasil: Fundamentos, situação atual e direções para o futuro. Psicologia: Teoria e Pesquisa, 26, 25-35. doi: 10.1590/ S0102-37722010000500003

Rasmussen, C., \& Bisanz, J. (2005). Representation and working memory in early arithmetic. Journal Experimental Child Psychology, 91, 137-157. doi: 10.1016/j.jecp.2005.01.004

Ribeiro, F. S., \& Santos, F. H. (2012). Treino musical e capacidade da memória operacional em crianças iniciantes, veteranas e sem conhecimentos musicais. Psicologia: Reflexão e Crítica, 25(3), 559-567. doi: 10.1590/S010279722012000300016

Ricker, T. J., Cowan, N., \& Morey, C. C. (2010). Visual working memory is disrupted by covert verbal retrieval. Psychonomic Bulletin \& Review, 17(4), 516-21. doi:10.3758/PBR.17.4.516

Rienstra, A., Spaan, P. E. J., \& Schmand, B. (2010). Validation of symptom validity tests using a "child-model" of adult cognitive impairments. Archives of Clinical Neuropsychology: The Official Journal of the National Academy of Neuropsychologists, 25(5), 371-82. doi:10.1093/arclin/acq035

Rodrigues, A., \& Befi-lopes, D. M. (2009). Memória operacional fonológica e suas relações com o desenvolvimento da linguagem infantil. Pró-Fono Revista de Atualização Científica, 21(1), 63-68. doi: 10.1590/S010456872009000100011

Rohde, T. E., \& Thompson, L. A. (2007). Predicting academic achievement with cognitive ability. Intelligence, 35(1), 83-92. doi:10.1016/j.intell.2006.05.004

Skogan, A. H., Zeiner, P., Egeland, J., Rohrer-Baumgartner, N., Urnes, A. G., Reichborn-Kjennerud, T., \& Aase, H. (2013). Inhibition and working memory in young preschool children with symptoms of ADHD and/or oppositional-defiant disorder. Child Neuropsychology, 20(5), 607-624. doi: 10.1080/09297049.2013.838213

Sternberg, R. J. (2009). Psicologia cogntiva (5 $5^{\text {Ed. }}$.). Cengage Learning.

Swanson, L. H. (2011). Intellectual growth in children as a function of domain specific and domain general working memory subgroups. Intelligence, 39(6), 481-492. doi:10.1016/j.intell.2011.10.001

Talarowska, M., Zboralski, K., \& Gałecki, P. (2013). Correlations between working memory effectiveness and depression levels after pharmacological therapy. Psychiatria Polska, 47(2), 255-267. Recuperado de http://www.psychiatriapolska.pl/uploads/images/PP_2_2013/ ENGverTalarowskaPP2_2013.pdf

Teixeira, R. A. A., Zachi, E. C., Roque, D. T., Taub, A., \& Ventura, D. F. (2011) Memory span measured by the spatial span tests of the Cambridge Neuropsychological Test Automated Battery in a group of Brazilian children and adolescents. Dementia Neuropsychology, 5(2), 129-134. Recuperado de http://www.demneuropsy.com.br/detalhe_artigo.asp?id=277

Tucker, A. M. (2013). Two independent sources of short-term memory problems during sleep deprivation. Sleep, 36(6), 815-7. doi: 10.5665/sleep.2696
Vallar, G., \& Baddeley, A. D. (1987). Phonological short-term store and sentence processing. Cognitive Neuropsychology, 4(4), 417-438. doi: 10.1080/02643298708252046

Vaz, I. A., Cordeiro, P. M., Macedo, E. C., \& Lukasova, K. (2010). Memória de trabalho em crianças avaliada pela tarefa de Brown-Peterson. Pró-Fono Revista de Atualização Científica, 22(2), 95-100. doi: 10.1590/S0104 56872010000200005

Wang, S., \& Gathercole, S. E. (2013). Working memory deficits in children with reading difficulties: Memory span and dual task coordination. Journal Experimental Child Psychology, 115(1), 188-97. doi: 10.1016/j. jecp.2012.11.015

Wechsler, D. (1997). Manual for the Wechsler Intelligence Scale for children-revised. Nova Iorque: Harcourt Test Publishers.

Zheng, X., Swanson, H. L., \& Marcoulides, G. A. (2011).Working memory components as predictors of children's mathematical word problem solving. Journal of Experimental Child Psychology, 110, 481-498. doi: 10.1016/j. jecp.2011.06.001 
Gustavo Marcelino Siquara, Mestre em Psicologia pela Universidade Federal da Bahia, é Professor Substituto na Universidade Estadual da Bahia e Professor Assistente na Escola Bahiana de Medicina e Saúde Pública. Endereço para correspondência: Laboratório de Pesquisa em Neuropsicologia Clinica e Cognitiva (NEUROCLIC), Av. Ademar de Barros, s/n - Pavilhão 04, Campus Universitário de Ondina Salvador/BA, CEP: 40170-110. Telefone: (71) 3283-6486. E-mail: siquara.gm@gmail.com

Maria Virgínia Machado Dazzani, Doutora em Educação pela Universidade Federal da Bahia, é Professora Adjunta do Instituto de Psicologia da Universidade Federal da Bahia. E-mail: dazzani@superig.com.br

Neander Abreu, Doutor em Neurociências e Comportamento pela Universidade de São Paulo, Pós-doutor pela Université du Luxembourg e The University of York, é Professor Adjunto do Instituto de Psicologia da Universidade Federal da Bahia. E-mail: neandersa@hotmail.com 